\title{
Antenna-Array Network Model
}

\author{
Supun De Silva, Michal Okoniewski, Fellow, IEEE, and Leonid Belostotski, Senior Member, IEEE
}

\begin{abstract}
An antenna can be modeled as a two-port network using S-parameters. However, having individual antenna Sparameters is not sufficient for modeling an array of antennas as antennas exhibit mutual coupling. This article proposes a network model to represent an antenna array with mutual coupling. Using the proposed model, an $N$-element antenna array is modeled as a combination of two-port networks representing each element and an $N$-port network representing mutual coupling. Two techniques to calculate mutual coupling $S$-parameters are presented: a primary method using numerical solutions and an approximate method using approximate expressions. The proposed model was validated using four monopole arrays having different interelement spacings, the number of elements, and non-identical antennas.
\end{abstract}

Index Terms-Antenna array, mutual coupling, S-parameters, radiation efficiency

\section{INTRODUCTION}

A NTENNA arrays are widely used across many fields: from radars [1]-[4] to mobile communication systems [5]-[10] to radio astronomy [11]-[15]. They offer many advantages over single antennas: high gain, high directivity, and the capability to steer the beam [16], [17]. However, arrays are more complex and more difficult to design due to mutual coupling than individual antennas [18].

Mutual coupling, due to the interchange of energy between array elements [16], alters the intended performance (e.g., the radiation pattern, beamwidth, input impedance, gain, and directivity) of each antenna element [19]-[21] and needs to be taken into account during array design. In addition it has been shown that mutual coupling affects array noise temperature [22]-[26]. This effect of mutual coupling on array noise performance has motivated this work. The discussions in this work do not deal with modeling the array beam pattern and it is assumed that the array is designed to meet its array-pattern requirements.

As was shown in [22]-[25], if the front-end amplifier, i.e. the low-noise amplifier (LNA), of a receiving antenna array is designed such that its optimum reflection coefficient for minimum noise, $\Gamma_{o p t}$, equals the antenna-array optimum reflection coefficient, $\Gamma_{a c t}$, then the array noise is minimized. While $\Gamma_{\text {opt }}$ can be measured [27], [28], $\Gamma_{a c t}$ is calculated based on the array electrical parameter and beamformer coefficients. The electrical parameters (e.g., S-parameters) in this case are associated with the physically accessible ports only. This type of modeling the array is not conducive to co-simulations with LNAs. Since the input signal arrives to the array from free space, there is a need for a "free-space" port to supply the

S. De Silva, M. Okoniewski and L. Belostotski are with the Department of Electrical and Computer Engineering, University of Calgary, AB, T2N 1N4 Canada.

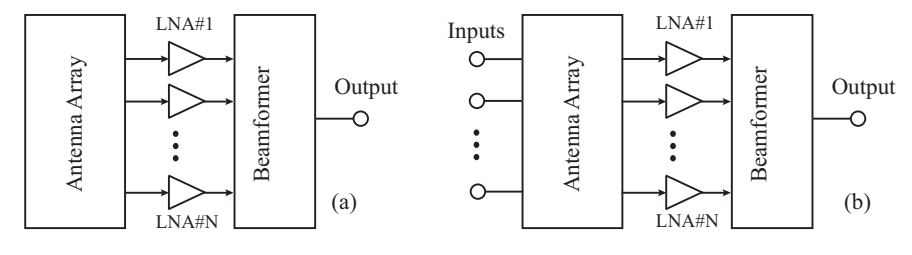

Fig. 1. Array modeling (a) conventional and (b) proposed.

input signal to the array so that in a circuit simulator a true codesign of the array and the active circuitry can be conducted. If such a model could be created and measured, then antenna arrays and LNAs could be fully co-designed. Fig. 1 shows the difference between the current way of modeling arrays and the proposed way. The beamformers in Fig. 1 are used to steer the array beams by scaling and combining individual antenna outputs. As such beamformers do not affect the antenna-array network model, they are not discussed in this work.

While much of the literature has been devoted to modeling antenna arrays using electrical parameters, e.g., Z-parameters or S-parameters, associated only with the physically accessible ports of the array [16], [17], [29]-[32], antenna arrays have also been analyzed as multi-port structures with some of the ports representing the radiated field patterns [33]. In this work we are interested in modeling and measuring an antenna array as an $2 N$ port network with $N$ ports associated with each of the $N$ antennas and the other $N$ ports representing the dominant far-field pattern that is associated with the dominant plane wave received by the array. If there are other modes generated by the antenna, but they are not associated to the incoming plane wave, they appear as antenna loss.

In addition to the possibility of co-designing receivers and antenna arrays, other properties of antenna arrays can be obtained with such a network. These include the radiation efficiency and its dependence on different excitations, matchingnetwork design with non-foster or passive components [34], array feed-network optimization, and even matching of the array to free space and its dependence on antenna design and termination.

Current literature supports modeling a single antenna using a two-port network S-parameters, which is explained in Section II. However, currently there seems to be no published work, apart from the authors' short explorations on this subject [35], [36], available on the network representation of an antenna array, which include all ports: physically accessible ports and the ports associated with the free space. This article attempts to fill this research gap.

\section{Two-Port NETWORK REPRESENTATION OF AN ANTENNA}

Fig. 2(a) presents an antenna and shows two of its ports. 
(a)

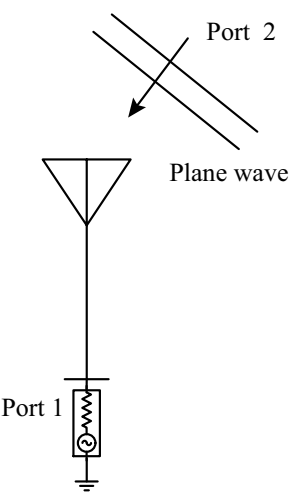

(b) $\stackrel{5}{=}$

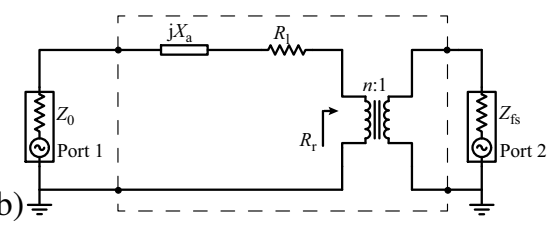

Fig. 2. (a) Single antenna represented with two-ports. (b) Two-port network representation of an antenna with the free-space impedance at Port 2

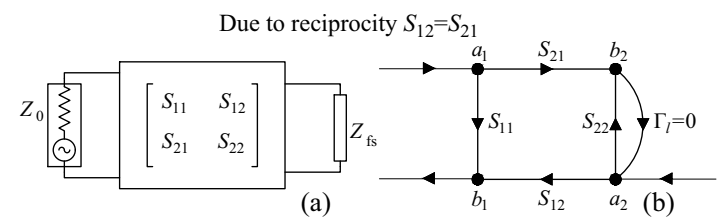

Fig. 3. (a) Two port representation and (b) signal-flow graph of an antenna modeled as a two-port network.

Port 1 is the conventional physically accessible port with the characteristic impedance $Z_{0}$ that connects antenna to a coaxial cable. Port 2 is a port referred to the free-space location where radiated waveform is considered a plane wave. The antenna in Fig. 2(a) can be represented as in Fig. 2(b) using a lumped frequency-dependent model of its dissipative loss resistance $\left(R_{l}\right)$, reactance $\left(X_{a}\right)$, and radiation resistance $\left(R_{r}\right)$ according to Thevenin's equivalent [16]. As the power delivered to the radiation resistance of an antenna represents the power delivered to the free space [34], an ideal transformer models impedance transformation from $R_{r}$ to the free space impedance $\left(Z_{f s}\right)$ [34]. With two ports defined, the standard two-port electrical parameters can be assigned to such an antenna by replacing the region marked by a dashed rectangle in Fig. 2(b) by a two-port network, for example, modeled with S-parameters as in Fig. 3(a).

For such a model, $S_{21}$ represents the power transfer from the connecting end of the cable to the radiation resistance and the free space in transmit mode. When the antenna is in receiving mode, $S_{12}$ denotes the power transfer through the antenna from free space to the radiation resistance and ultimately to Port $1 . S_{22}$ denotes the back reflection occurring when the power is delivered from the radiation resistance in receiving mode. $S_{11}$ is the reflection coefficient of Port 1 and is the only parameter readily measurable with a vector network analyzer (VNA). Due to reciprocity [17], $S_{21}=S_{12}$, which was used throughout this article.

Fig. 3(b) presents the signal-flow graph of an antenna modeled as a two-port network. The free space impedance $Z_{\mathrm{fs}}$ is modeled with $\Gamma_{l}=0$ as it represents a matched termination [37]. It should also be noted that Port 1 and Port 2 have different characteristic impedances.

With the two-port network of an antenna as described,

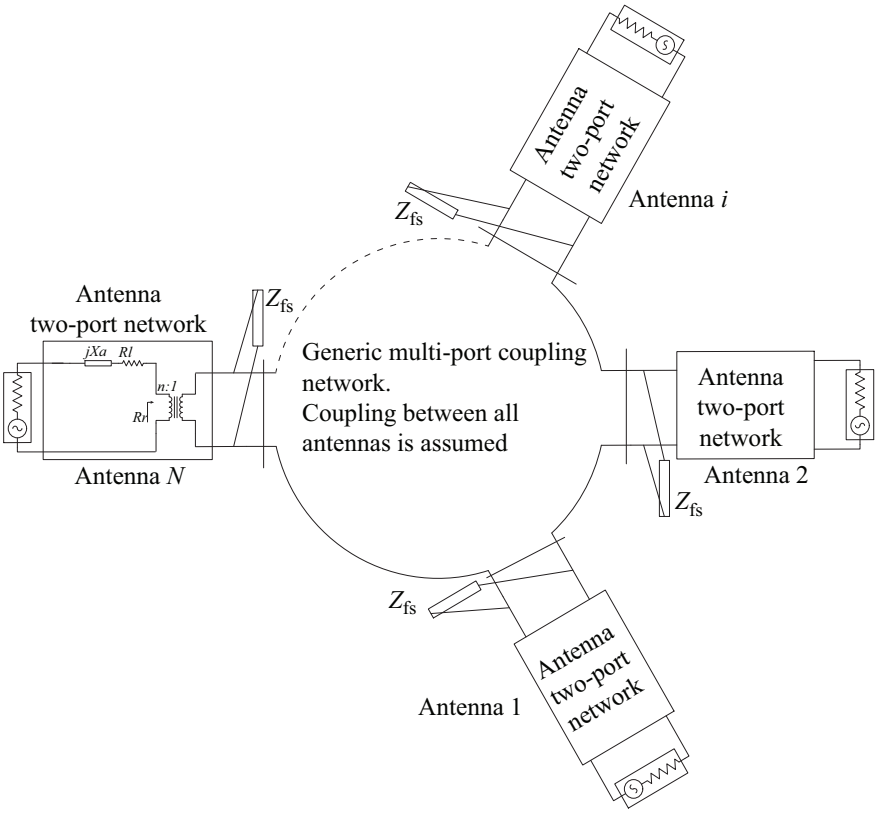

Fig. 4. Schematic diagram of an $N$-element antenna array.

its two-port S-parameter can be measured by either of the following three methods:

- if the radiation efficiency of an antenna is known or readily available, the method presented by Aberle in [34] can be used.

- if the radiation efficiency of an electrically small antenna is unknown, the radiation efficiency can be calculated using the IWC method presented by Johnston et al. in [37]. Subsequently, the two-port S-parameters can be determined by the method presented by Aberle in [34].

- if the radiation efficiency of an electrically small antenna is unknown and it radiates spherical waves, two-port Sparameters can be determined by SWC method presented by Thal in [38] and [39], [40].

In this work, the last of the three approaches is used.

\section{Representation of Mutual Coupling as a Multi-Port Device}

This article proposes to model a passive, linear antenna array using a combination of two-port antenna networks and a multi-port free-space-referred mutual-coupling network, which aggregates all coupling modes. S-parameters of the multi-port model are denoted as $\mathbf{S}_{\mathrm{Mu}}$. The number of ports of the multiport network equals to the number of antenna elements of the array. Fig. 4 presents the schematic diagram of the $N$-element antenna array, where its signal-flow graph is presented in Fig. 5.

The following two sections discuss two proposed methods of determining the elements of $\mathbf{S}_{\mathrm{Mu}}$ matrix: a method termed "Primary," which does not introduce any simplifying assumptions, and a method termed "Approximate," which simplifies expressions by neglecting re-scattering of $S_{22}$ of neighboring antennas and permits analytical solution for $\mathbf{S}_{\mathrm{Mu}}$. 


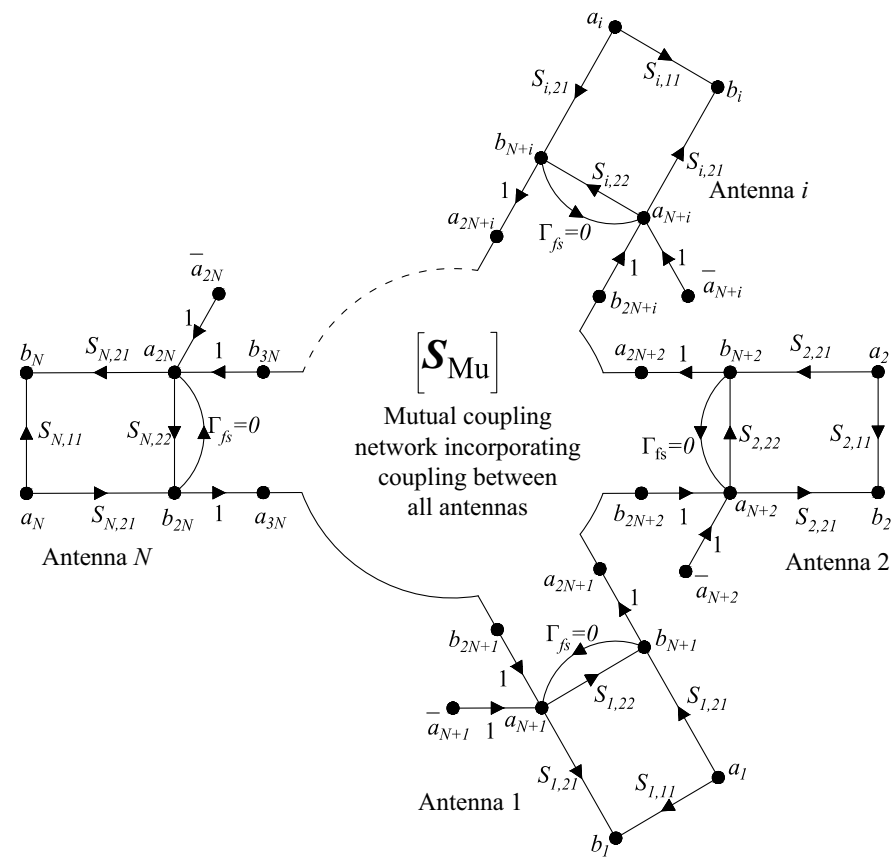

Fig. 5. Signal-flow graph of an $N$-element antenna array. The wave transmitted to the free space for antenna $i$ is associated with node $b_{N+i}$ and the incident wave $\bar{a}_{N+i}$ from the free space arrives to node $a_{N+i}$. Mutual coupling is modeled by travelling waves flowing from node $b_{N+i}$ to node $a_{2 N+i}$ and from node $b_{2 N+i}$ to node $a_{N+i}$.

\section{A. Primary Method of finding $\mathbf{S}_{\mathrm{Mu}}$}

Since mutual coupling occurs through the free space in near and far fields and cannot be measured directly as the ports of the modeled muti-port network are not physically accessible by a VNA, the elements of $\mathbf{S}_{\mathrm{Mu}}$ have to be calculated from results of other measurements. Here, the antenna S-parameters are measured as in Section II and a VNA is used to measure S-parameters $\mathbf{S}_{\mathrm{VNA}}$ of the physically accessible ports 1 to $N$, which relate to the traveling waves $a_{i}$ and $b_{j}$ in Fig. 5 by [41]

$$
S_{\mathrm{VNA}, i i}=\left.\frac{b_{i}}{a_{i}}\right|_{a_{x}=0 \text { for } x \neq i, \bar{a}_{N+1 \ldots 2 N}=0}
$$

and

$$
S_{\mathrm{VNA}, i j}=\left.\frac{b_{i}}{a_{j}}\right|_{a_{x}=0 \text { for } x \neq j, \bar{a}_{N+1 \ldots 2 N}=0},
$$

where $x, i, j \in \mathbb{N}$ and $1 \leq x, i, j \leq N$.

There exists $N^{2}$ (less due to reciprocity) of S-parameter measurements that can be derived according to (1) and (2) using a VNA. As each port is exited by $a_{i}$, the portion of $a_{i}$ is delivered to the free-space ports and a portion propagates through the network in Fig. 5 to a measurement port. We then use the measured 2-port S-parameters of each antenna (see Section II) and the measured matrix $\mathbf{S}_{\mathrm{VNA}}$ and (1) and (2) to solve for the elements of $\mathbf{S}_{\mathrm{Mu}}$ using numerical methods. Examples of using this methods for arrays of two antennas was presented by the authors in [35], [36] and briefly discussed next.

Calculation of $\mathbf{S}_{\mathrm{Mu}}^{\mathrm{Pri}}$ for a 2-Antenna Array: For a 2antenna array, represented with networks in Fig. 6, the node expressions are

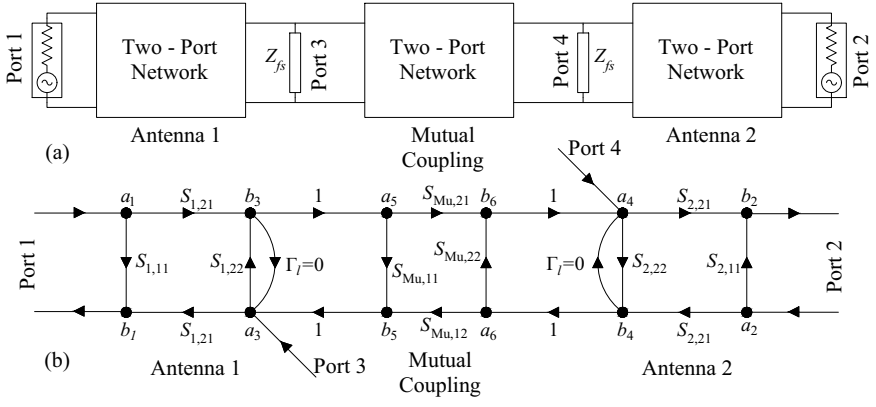

Fig. 6. (a) Schematic and (b) signal-flow graph of a 2-antenna array.

$$
\begin{aligned}
& b_{1}=S_{1,11} a_{1}+S_{1,21} a_{3}, \\
& b_{2}=S_{2,21} a_{4}+S_{2,11} a_{2}, \\
& b_{3}=S_{1,22} a_{3}+S_{1,21} a_{1}, \\
& b_{4}=S_{2,22} a_{4}+S_{2,21} a_{2}, \\
& b_{5}=S_{\mathrm{Mu}, 11} a_{5}+S_{\mathrm{Mu}, 12} a_{6}, \\
& b_{6}=S_{\mathrm{Mu}, 21} a_{5}+S_{\mathrm{Mu}, 22} a_{6} \\
& a_{3}=b_{5}, a_{4}=b_{6} \\
& a_{5}=b_{3}, a_{6}=b_{4} .
\end{aligned}
$$

where $\bar{a}_{N+i}, 1 \leq i \leq N$, are set to zero since there are no incident waves to the free-space ports during VNA measurements.

These expressions are solved for $b_{1, \ldots, 6}$ in terms of $a_{1,2}$, known S-parameters of each array antenna and unknown $\mathbf{S}_{\mathrm{Mu}}$. In this work, 'solve' function in MATLAB was used to perform the computation [42]. Then, expressions for $S_{\mathrm{VNA}, 11}$, $S_{\mathrm{VNA}, 22}, S_{\mathrm{VNA}, 12}$, and $S_{\mathrm{VNA}, 21}$ as defined in (1) and (2) are obtained and equated to the VNA measurements of the same. At that stage, the only unknowns are the elements of $\mathbf{S}_{\mathrm{Mu}}$, which are found in MATLAB using the function 'fsolve' [43].

\section{B. Approximate Method of finding $\mathbf{S}_{\mathrm{Mu}}$}

The method of finding $\mathbf{S}_{\mathrm{Mu}}$ explained in Section III-A applies for large arrays but becomes computationally involved. However, it can be simplified without introducing significant errors as is shown in Section V with measurement results.

To simplify the signal-flow graph and the subsequent calculations, reflections of $S_{x, 22}$ where $x \neq i$ are neglected, thereby assuming that the energy re-scattered from other antennas is approximately zero. Then (1) and (2) become

$$
\begin{aligned}
& S_{\mathrm{VNA}, i i} \approx \tilde{S}_{i i}=\frac{b_{i}}{a_{i}} \mid \begin{array}{l}
a_{x}=0, S_{x, 22} \approx 0 \text { for } x \neq i \\
a_{N+1 \ldots 2 N}=0
\end{array} \\
& S_{\mathrm{VNA}, i j} \approx \tilde{S}_{i j}=\frac{b_{i}}{a_{j}} \mid \begin{array}{l}
a_{x}=0, S_{y, 22} \approx 0 \text { for } x \neq j \& y \neq i, j \\
a_{N+1 \ldots 2 N}=0
\end{array}
\end{aligned}
$$

where $x, y, i, j \in \mathbb{Z}$ and $1 \leq x, y, i, j \leq N$. This simplification avoids signal-flow paths created due to multiple re-scattering from other antennas, which typically are very low in strength. 
Thus, the approximation is not expected to significantly impact the numerical solution but significantly reduces the complexity of the signal-flow graph. The rest of the method is the same as in Section III-A and results in $\mathbf{S}_{\mathrm{Mu}}^{\mathrm{App}}$. However, using mathematical induction, approximate expressions for mutual coupling of S-parameters considering the $i^{\text {th }}$ element and the $j^{\text {th }}$ element in an $N$-antenna array are inferred as

$$
S_{\mathrm{Mu}, i i}^{\mathrm{App}}=\frac{S_{i, 11}-\tilde{S}_{i i}}{S_{i, 22}\left(S_{i, 11}-\tilde{S}_{i i}\right)-S_{i, 21}^{2}}
$$

and

$$
S_{\mathrm{Mu}, i j}^{\mathrm{App}}=\mathbb{X} \sqrt{\frac{\mathbb{J}+\mathbb{M}+\mathbb{P Y}}{\mathbb{Y} \mathbb{H}}}-\mathbb{X}
$$

where

$$
\begin{aligned}
\mathbb{X} & =\frac{S_{i, 21} S_{j, 21}}{2 S_{i, 22} S_{j, 22} \tilde{S}_{i j}}, \\
\mathbb{Y} & =\left(S_{i, 21}^{2}+S_{i, 22} \tilde{S}_{i i}-S_{i, 11} S_{i, 22}\right), \\
\mathbb{H} & =\left(S_{j, 21}^{2}+S_{j, 22} \tilde{S}_{j j}-S_{j, 11} S_{j, 22}\right), \\
\mathbb{J} & =S_{j, 21}^{2}\left(S_{i, 21}^{2}-S_{i, 11} S_{i, 22}\right), \\
\mathbb{M} & =S_{i, 22}\left(4 S_{j, 22} \tilde{S}_{i j}^{2}+S_{j, 21}^{2} \tilde{S}_{i i}\right), \\
\mathbb{P} & =S_{j, 22}\left(\tilde{S}_{j j}-S_{j, 11}\right) .
\end{aligned}
$$

Calculation of $\mathbf{S}_{\mathrm{Mu}}^{\mathrm{App}}$ for 2-Antenna Array: In the approximate method, the procedure of finding $\mathbf{S}_{\mathrm{Mu}}^{\mathrm{App}}$ is the same as for finding $\mathbf{S}_{\mathrm{Mu}}^{\mathrm{Pri}}$ and follows the array representation in Fig. 6 and the node expressions in (3) but with re-scattering off the neighboring antennas ignored.

\section{Complete Scattering Matrix of AN Antenna ARRAY}

Complete scattering parameters of an antenna array $\left(\mathbf{S}_{\mathrm{Ar}}\right)$ present the relationship between all ports associated with array elements. The signal-flow graph of an $N$-antenna array is presented in Fig. 5, where the incident traveling waves on the free-space port $i$ is represented by $\bar{a}_{N+i}$. With $\mathbf{S}_{\mathrm{Mu}}$ found with the either the method in Sections III-A or III-B and 2port antenna S-parameters known from Section II, all elements of the network in Fig. 5 are known from which a multi-port network model of the array

$$
\left(\begin{array}{c}
b_{1} \\
\vdots \\
b_{N} \\
b_{N+1} \\
\vdots \\
b_{2 N}
\end{array}\right)=\mathbf{S}_{\mathrm{Ar}}\left(\begin{array}{c}
a_{1} \\
\vdots \\
a_{N} \\
\bar{a}_{N+1} \\
\vdots \\
\bar{a}_{2 N}
\end{array}\right)
$$

in Fig. 7 is readily obtained, where ports from 1 to $N$ are the physically accessible ports of each antenna and ports $N+1$ to $2 N$ are associated with the free space.

Since $\mathbf{S}_{\mathrm{Mu}}$ can be calculated using the primary method or the approximate method, $\mathbf{S}_{\mathrm{Ar}}$ can also be calculated using $\mathbf{S}_{\mathrm{Mu}}^{\mathrm{Pri}}$ or $\mathbf{S}_{\mathrm{Mu}}^{\mathrm{App}}$, resulting in $\mathbf{S}_{\mathrm{Ar}}^{\mathrm{Pri}}$ and $\mathbf{S}_{\mathrm{Ar}}^{\mathrm{App}}$.

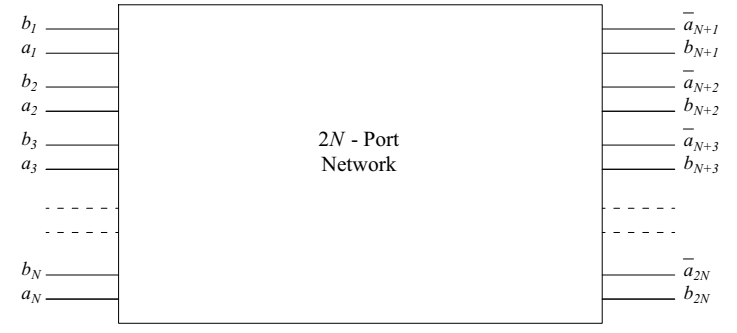

Fig. 7. Schematic diagram of an $N$-element antenna array represented with an $2 N$-port network.

\section{MOdEl Verification}

This section presents the measurements, calculations, and simulation results, which are used to validate the proposed model. Since all antennas used in the verification are electrically small single-mode monopole antennas, whose Sparameters are measured using methods in [38]-[40], the applicability of this method to other types of antennas has not been confirmed. Four different monopole arrays are tested to validate the model. For each array, the radiation efficiency and the S-parameters of the physically accessible ports are calculated using both $\mathbf{S}_{\mathrm{Mu}}^{\mathrm{Pri}}$ and $\mathbf{S}_{\mathrm{Mu}}^{\mathrm{App}}$. Subsequently, the results are compared with simulation results and measurements for accuracy assessment. The parameters that are compared include:

1) calculated input reflection coefficients of the array from the proposed model, which can be directly compared to the VNA measurements. It is expected that since the calculation of $\mathbf{S}_{\mathrm{Ar}}^{\mathrm{Pri}}$ is based on the data from VNA measurements and since there are no simplifying assumptions in the calculations, reflection coefficients found using $\mathbf{S}_{\mathrm{Ar}}^{\mathrm{Pri}}$ would perfectly match VNA measurements. However, $\mathbf{S}_{\mathrm{Ar}}^{\mathrm{App}}$ matrix may show some discrepancy.

2) calculated efficiency of the array, which is compared to HFSS simulated efficiency, where according to [44] the radiation efficiency of an antenna is defined as

$$
\text { Radiation Efficiency }\left(\eta_{\text {Ar }}\right)=\frac{\text { Radiated Power }}{\text { Net Accepted Power }}
$$

or equivalently

$$
\eta_{\mathrm{Ar}}=\frac{\sum_{i=1}^{N}\left(\left|b_{N+i}\right|^{2}-\left|a_{N+i}\right|^{2}\right)}{\sum_{i=1}^{N}\left(\left|a_{i}\right|^{2}-\left|b_{i}\right|^{2}\right)},
$$

where power at each port is calculated according to [45]. In order to determine the radiation efficiency using (10), the signal-flow graph is used to find the node expressions for reflected power wave amplitudes $\left(b_{i}\right)$ in terms of incident power wave amplitudes $\left(a_{i}\right)$.

It should be also noted that the simulated radiation efficiencies of the arrays discussed next were over $100 \%$ with default HFSS meshing. Similar behavior was noted in the past for example in [23], [46]. To improve accuracy, the maximum element length of the mesh was set to $\lambda / 15$ in this study. 


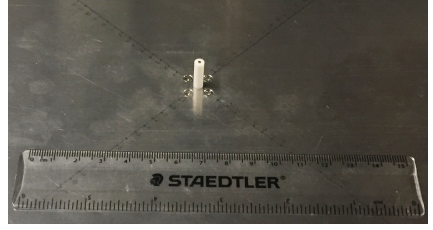

Fig. 8. The prototype of single monopole on a ground plane.

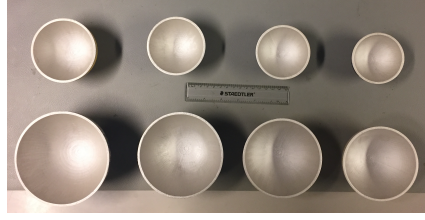

(a) Top view.

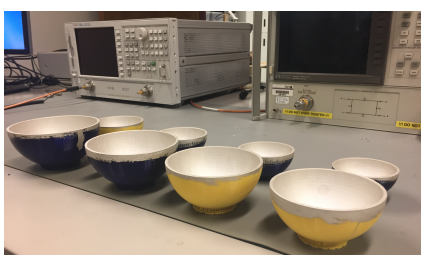

(b) Side view.
Fig. 9. 3D printed caps painted with metallic silver paint.

Further decreasing the maximum element length of the mesh could slightly increase the accuracy but extremely increase the simulation time. Therefore, it is possible that the HFSS radiation-efficiency results in the following sections might be slightly overestimated.

To model various antenna arrays in Sections V-B to V-D, first 2-port S-parameters of a single antenna are required and are discussed next.

\section{A. S-Parameters of a Single Array Element: Monopole}

All arrays discussed in this work are fabricated using identical monopoles in Fig. 8. Each monopole is made of copper (height: $14 \mathrm{~mm}$, diameter: $1.27 \mathrm{~mm}$ ) and is surrounded by a cylinder of Teflon (relative permittivity: 2.1, height: 14 $\mathrm{mm}$, inner diameter: $1.27 \mathrm{~mm}$, outer diameter: $4.11 \mathrm{~mm}$ ). An aluminum sheet (length: $380 \mathrm{~mm}$, width: $380 \mathrm{~mm}$, thickness: $1.14 \mathrm{~mm}$ ) is used as the ground plane. The frequency points of interest are chosen to be $4.2 \mathrm{GHz}, 4.6 \mathrm{GHz}, 5.0 \mathrm{GHz}$, and $5.4 \mathrm{GHz}$.

In order to calculate two-port $\mathbf{S}_{i}$ of a single monopole antenna, the SWC method is used [38], [40]. Spherical Wheeler cap radii are calculated according to [38], [40] and were 3D printed. Their inner surfaces were painted with conductive metallic silver paint. The fabricated caps are presented in Fig. 9. It should be noted that the metallic cap, the ground plane, and the contact between the metallic cap and the ground plane introduce some additional loss [38], [47] that may not be fully accounted for in simulations.

\section{B. Arrays with Different Antenna Spacings}

In order to test the performance of the proposed model as a function of inter-element distance $(d)$ and hence mutual coupling, two 2-element arrays with identical monopole antennas and $d=30 \mathrm{~mm}$ and $40 \mathrm{~mm}$ were built and shown in Fig. 10.

Measured radiation efficiency of the antenna array was calculated according to (10) and compared to the efficiency simulated with HFSS in Figs. 11(a) and 12(a). It can be observed that the simulation results and calculated results using measured $\mathbf{S}_{\mathrm{Ar}}^{\mathrm{Pri}}$ or $\mathbf{S}_{\mathrm{Ar}}^{\mathrm{App}}$ are very close to each other, thus

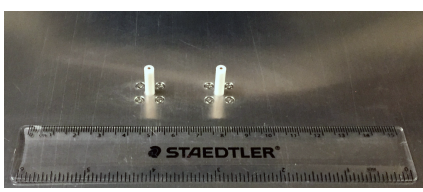

(a) $d=30 \mathrm{~mm}$

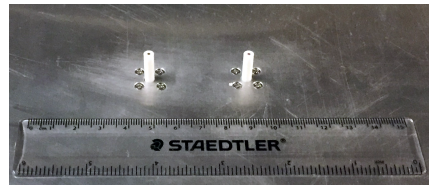

(b) $d=40 \mathrm{~mm}$
Fig. 10. Tested two-element arrays with different inter-element distances.
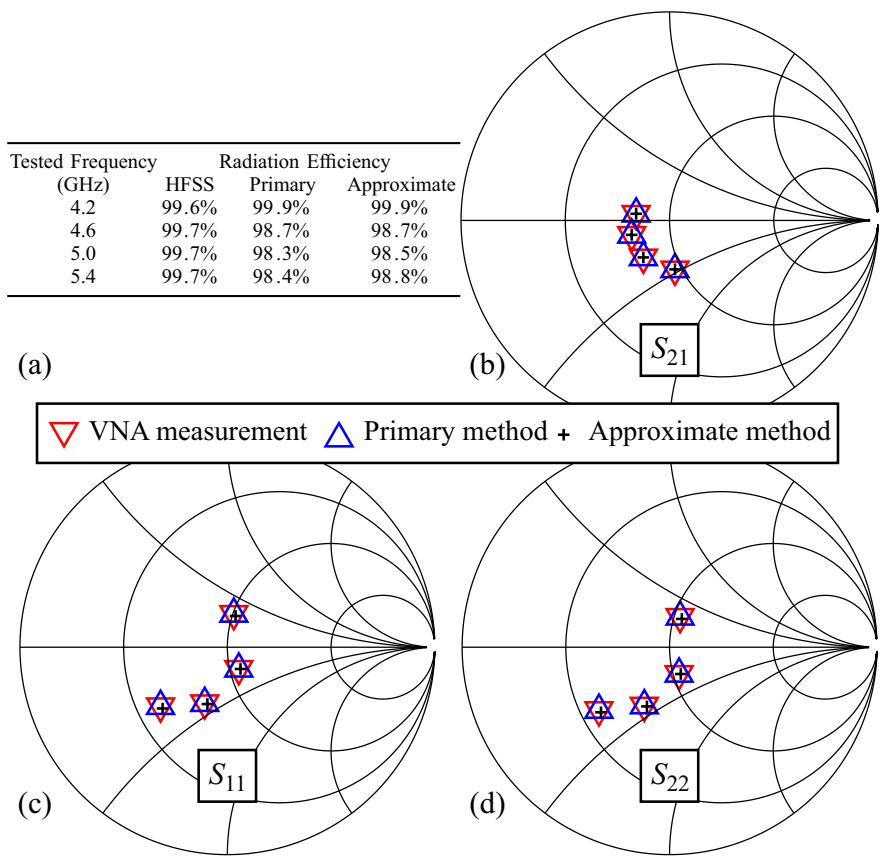

Fig. 11. Comparison between a) radiation efficiency of the measured array with $d=30 \mathrm{~mm}$ and that obtained using HFSS simulations and b)-d) Sparameters calculated from $\mathbf{S}_{\mathrm{Ar}}^{\mathrm{Pri}}$ and $\mathbf{S}_{\mathrm{Ar}}^{\mathrm{App}}$ and that obtained directly with the VNA.

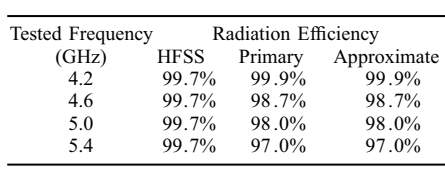

(a)

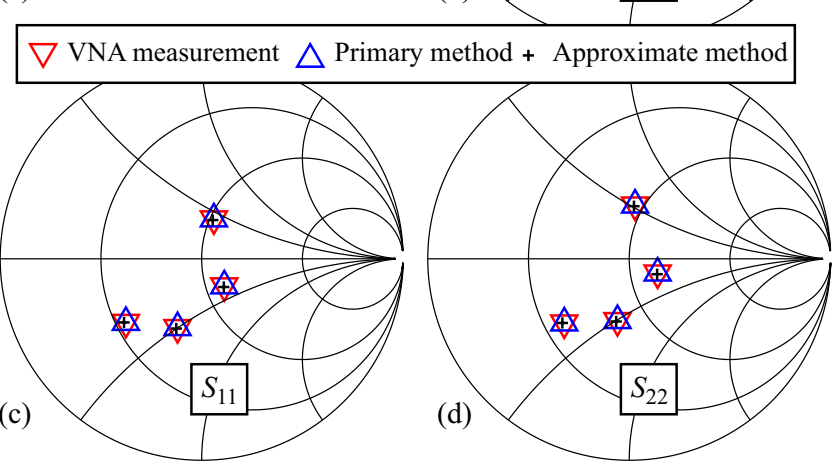

Fig. 12. Comparison between a) radiation efficiency of the measured array with $d=40 \mathrm{~mm}$ and that obtained using HFSS simulations and b)-d) Sparameters calculated from $\mathbf{S}_{\mathrm{Ar}}^{\mathrm{Pri}}$ and $\mathbf{S}_{\mathrm{Ar}}^{\mathrm{App}}$ and that obtained directly with the VNA. 


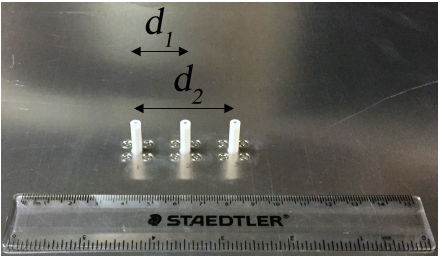

Fig. 13. Three-element linear antenna arrays. Antenna spacings are: $d_{1}=20$ $\mathrm{mm} d_{2}=40 \mathrm{~mm}$

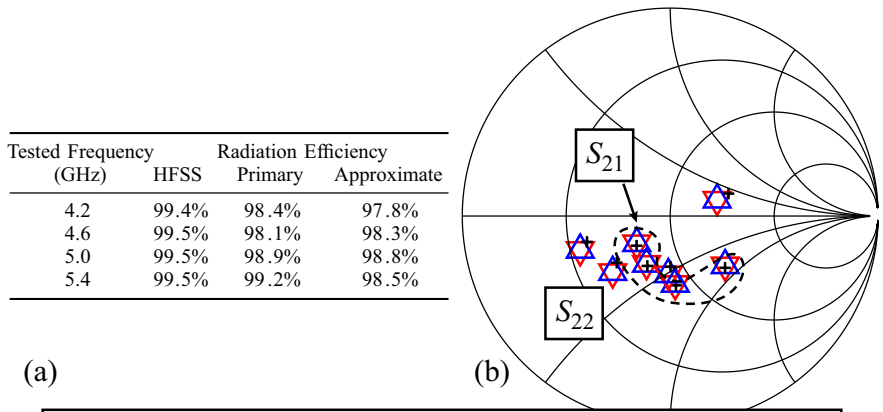

$\nabla$ VNA measurement $\triangle$ Primary method + Approximate method
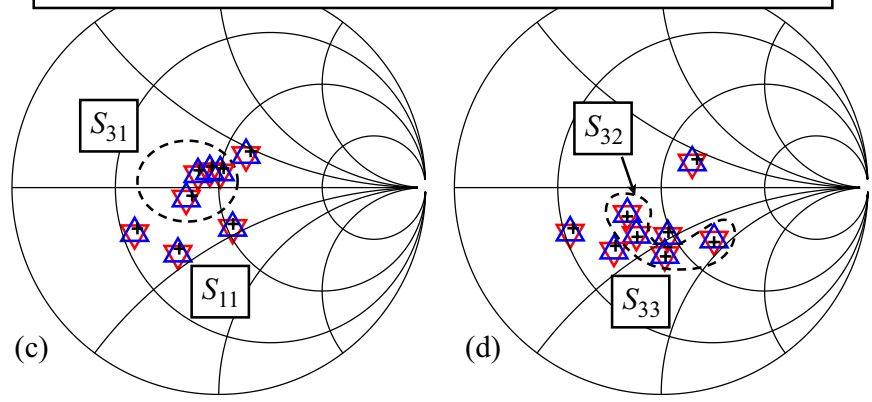

Fig. 14. Comparison between a) radiation efficiency of the measured 3-element array and that obtained using HFSS simulations and b)-d) Sparameters calculated from $\mathbf{S}_{\mathrm{Ar}}^{\mathrm{Pri}}$ and $\mathbf{S}_{\mathrm{Ar}}^{\mathrm{App}}$ and that obtained directly with the VNA.

demonstrating the ability of the proposed model to calculate the radiation efficiency of an array.

S-parameters of the physically accessible ports were found from the array models $\mathbf{S}_{\mathrm{Ar}}^{\mathrm{Pri}}$ or $\mathbf{S}_{\mathrm{Ar}}^{\mathrm{App}}$ for comparison to directly measured S-parameters using the VNA. Fig. 11(b)-(d) and Fig. 12(b)-(d) present the results. As expected, the primary method gives perfect agreement with the measurements, whereas the approximate method exhibits slight errors in $S_{11}, S_{22}$, and $S_{21}$.

\section{Three-Antenna Array}

The proposed model was also tested with a three-element linear array. This array was also fabricated using identical monopoles, already discussed in Section V-A, on a ground plane as shown in Fig. 13.

The calculated radiation efficiency of the array is shown in Fig.14(a) It can be observed that the simulation results and calculations using $\mathbf{S}_{\mathrm{Ar}}^{\mathrm{Pri}}$ and $\mathbf{S}_{\mathrm{Ar}}^{\mathrm{App}}$ are again similar to simulations.

As with the first two arrays, for this array the S-parameters of the physically accessible ports were calculated based on $\mathbf{S}_{\mathrm{Ar}}^{\mathrm{Pri}}$ and $\mathbf{S}_{\mathrm{Ar}}^{\mathrm{App}}$ for comparison with directly measured data. In

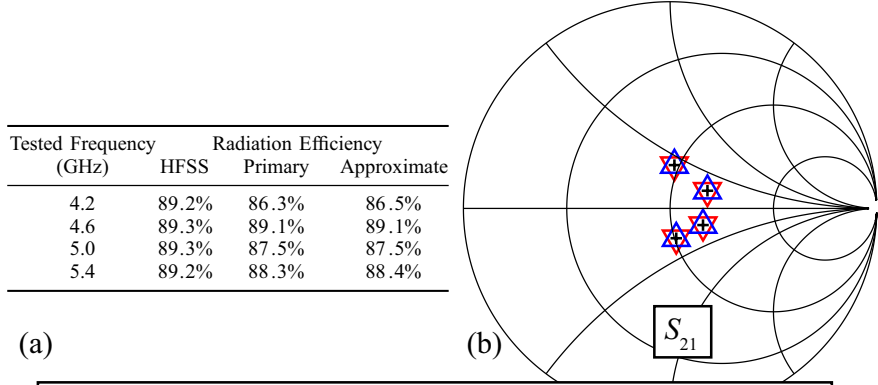

$\nabla$ VNA measurement $\triangle$ Primary method + Approximate method
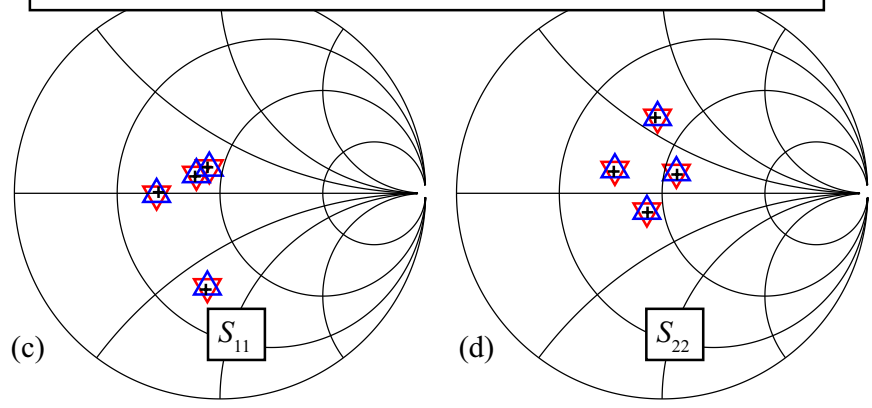

Fig. 15. Comparison between a) radiation efficiency of the measured array with one lossy antenna and that obtained using HFSS simulations and b)-d) S-parameters calculated from $\mathbf{S}_{\mathrm{Ar}}^{\mathrm{Pri}}$ and $\mathbf{S}_{\mathrm{Ar}}^{\mathrm{App}}$ and that obtained directly with the VNA.

this case, there are three such ports. The results are shown in Fig. 14(b)-(d). It is observed that the measured and calculated S-parameters are near identical for the tested three-element linear array with the largest deviations associated with the approximate method.

\section{Non-Identical Antennas in an Array}

To investigate whether the method is viable for arrays of non-identical antennas due to, for example, fabrication tolerances, this section considers an extreme scenario in which the two-element array with $d=30 \mathrm{~mm}$ was used while a 1$\mathrm{dB}$ attenuator was attached to the second element. The SWC method in Section V-A was repeated for the lossy antenna to obtain its two-port antenna S-parameters.

As above, the newly obtained $\mathbf{S}_{\mathrm{Ar}}^{\mathrm{Pri}}$ and $\mathbf{S}_{\mathrm{Ar}}^{\mathrm{App}}$ were used to calculate the array radiation efficiency and the S-parameters that are measurable by a VNA. The results are shown in Fig. 15.

As shown, the method is capable of treating non-equal antennas and the array radiation efficiency is sensitive to the array losses. It is noted that there are larger difference between simulated and calculated radiation efficiencies than what was seen for other arrays. The reason for this is that the attenuator insertion loss is frequency dependent but in the simulations it was set to a constant value.

\section{CONCLUSION}

This article has proposed, developed, and validated a $2 \mathrm{~N}$ network model for an $N$-element antenna array. In this model, $N$ ports are associated with free space and $N$ ports are the physically accessible antenna terminals. The model represents 
each of the $N$ antennas as a two-port network and mutual coupling between antennas as a $N$-port network. The article presented two methods for finding the model elements. One method is complete and provided better agreement than an approximate method. The advantage of the approximate method in the possibility of finding an analytical solution to the model.

In order to validate the proposed methods several monopole arrays were used: different inter-element spacings $(d=$ $30 \mathrm{~mm}, 40 \mathrm{~mm}$ ), different number of elements, and nonidentical array elements. The proposed array model was validated with reference to the radiation efficiency and the calculation of S-parameters that would be measured by a VNA.

It was observed that while the calculated radiation efficiency is sensitive to the VNA measurement and HFSS mesh size, the proposed model resulted in the efficiency that was typically within $2 \%$ of the simulated efficiency. Thus, it was concluded that the proposed model is validated.

\section{ACKNOWLEDGMENT}

The authors would like to thank the Applied Electromagnetics Group (AEG) and the Department of Electrical and Computer Engineering of the University of Calgary for all the resources and facilities provided to make this research a success. Special thanks goes out to Jeremie Bourqui for the assistance at the laboratory and providing all the resources. Also, the authors wish to extend their appreciation to Joe Sabourin at the machines shop for fabricating the antenna arrays.

In addition, the authors like to thank the CMC Microsystems for providing the necessary software tools.

\section{REFERENCES}

[1] W. D. Wirth, Radar techniques using array antennas, ser. Radar, sonar, navigation and avionics series. London: IET, 2001, no. 10.

[2] E. Brookner, "Phased array radars-past, present and future," in IET RADAR, Edinburgh, Oct. 2002, pp. 104-113.

[3] E. Brookner, "Developments and breakthroughs in radars and phasedarrays," in IEEE Radar Conference, Philadelphia, May 2016, pp. 1-6.

[4] D. B. Hayman, A. P. Chippendale, A. W. Hotan, R. D. Shaw, S. G. Hay, T. S. Bird, P. J. Hall, and K. P. Esselle, "Measuring radiotelescope phased array feed noise and sensitivity," in Eur. Conf. Antennas Propag., Apr. 2014, pp. 3526-3530.

[5] A. J. Fenn and P. T. Hurst, Ultrawideband phased array antenna technology for airborne and ground-based systems, ser. Lincoln Laboratory series. Cambridge, Massachusetts: The MIT Press, 2015.

[6] L. C. Godara, "Applications of antenna arrays to mobile communications. i. performance improvement, feasibility, and system considerations," Proc. IEEE, vol. 85, no. 7, pp. 1031-1060, Jul. 1997.

[7] J. Zhang, X. Ge, Q. Li, M. Guizani, and Y. Zhang, "5G Millimeter-wave antenna array: Design and challenges," IEEE Wirel. Commun., vol. 24, no. 2, pp. 106-112, Apr. 2017.

[8] S. Rangan, T. S. Rappaport, and E. Erkip, "Millimeter-wave cellular wireless networks: Potentials and challenges," Proc. IEEE, vol. 102, no. 3, pp. 366-385, Mar. 2014.

[9] W. Roh, J. Y. Seol, J. H. Park, B. Lee, J. Lee, Y. Kim, J. Cho, K. Cheun, and F. Aryanfar, "Millimeter-wave beamforming as an enabling technology for 5G cellular communications: Theoretical feasibility and prototype results," IEEE Commun. Mag., vol. 52, no. 2, pp. 106-113, Feb. 2014.

[10] T. S. Rappaport, F. Gutierrez, E. Ben-Dor, J. N. Murdock, Y. Qiao, and J. I. Tamir, "Broadband millimeter-wave propagation measurements and models using adaptive-beam antennas for outdoor urban cellular communications," IEEE Trans. Antennas Propag., vol. 61, no. 4, pp. 1850-1859, Apr. 2013.
[11] L. Lei, K. Grainge, and A. Navarrini, "Analysis of Vivaldi array antenna for phased array feeds application," in IEEE Int. Conf. Numer. Electromagn. Multiphysics Model. Optim. RF, Microwave, Terahertz Appl., Seville, May 2017, pp. 233-235.

[12] M. A. Garrett, "Radio astronomy transformed: aperture arrays - past, present and future," in IEEE Africon, Sep. 2013, pp. 1-5.

[13] H. Steyskal and J. Herd, "Mutual coupling compensation in small array antennas," IEEE Trans. Antennas Propag., vol. 38, no. 12, pp. 19711975, Dec. 1990.

[14] H. T. Friis and C. B. Feldman, "A multiple unit steerable antenna for short-wave reception," Proc. IRE, vol. 25, no. 7, pp. 337-419, Jul. 1937.

[15] P. Dewdney, P. Hall, R. Schilizzi, and T. Lazio, "The Square Kilometre Array," Proc. IEEE, vol. 97, no. 8, pp. 1482-1496, Aug. 2009.

[16] C. A. Balanis, Antenna theory analysis and design, 3rd ed. Hoboken, New Jersey: John Wiley \& Sons, Inc, 2005.

[17] W. L. Stutzman and G. A. Thiele, Antenna theory and design, 2nd ed. New York: J. Wiley, 1998.

[18] D. G. Fang, Antenna theory and microstrip antennas. Boca Raton, FL: CRC Press / Taylor \& Francis, 2010.

[19] I. Gupta and A. Ksienski, "Effect of mutual coupling on the performance of adaptive arrays," IEEE Trans. Antennas Propag., vol. 31, no. 5, pp. 785-791, Sep. 1983.

[20] H. Singh, H. L. Sneha, and R. M. Jha, "Mutual coupling in phased arrays: a review," Int J Antenn Propag, vol. 2013, pp. 1-23, Mar. 2013.

[21] J. A. G. Malherbe, "Analysis of a linear antenna array including the effects of mutual coupling," IEEE Trans. Educ., vol. 32, no. 1, pp. 2934, Feb. 1989.

[22] K. F. Warnick, B. Woestenburg, L. Belostotski, and P. Russer, "Minimizing the Noise Penalty Due to Mutual Coupling for a Receiving Array," IEEE Trans. Antennas Propag., vol. 57, no. 6, pp. 1634-1644, 2009.

[23] R. Maaskant, D. Bekers, M. Arts, W. van Cappellen, and M. Ivashina, "Evaluation of the radiation efficiency and the noise temperature of lowloss antennas," IEEE Antennas Wireless Propag. Lett., vol. 8, pp. 11661170, Oct. 2009.

[24] K. F. Warnick, M. V. Ivashina, R. Maaskant, and B. Woestenburg, "Unified definitions of efficiencies and system noise temperature for receiving antenna arrays," IEEE Trans. Antennas Propag., vol. 58, no. 6, pp. 2121-2125, jun 2010

[25] L. Belostotski, B. Veidt, K. F. Warnick, and A. Madanayake, "Low-noise amplifier design considerations for use in antenna arrays," IEEE Trans. Antennas Propag., vol. 63, no. 6, pp. 2508-2520, Jun 2015.

[26] E. Woestenburg, R. Witvers, M. Ruiter, and P. Benthem, "Improved sensitivity of a low noise aperture array tile for the SKA," in Int. Conf. Electromagn. Adv. Appl. IEEE, Aug. 2014, pp. 147-150.

[27] L. Belostotski and J. W. Haslett, "Evaluation of tuner-based noiseparameter extraction methods for very low noise amplifiers," IEEE Trans. Microw. Theory Tech., vol. 58, no. 1, pp. 236-250, January 2010.

[28] M. Himmelfarb and L. Belostotski, "On impedance-pattern selection for noise parameter measurement," IEEE Transactions on Microwave Theory and Techniques, vol. 64, no. 1, pp. 258-270, Jan 2016.

[29] R. J. Mailloux, Phased array antenna handbook, 2nd ed., ser. Artech House antennas and propagation library. Boston: Artech House, 2005.

[30] H. J. Visser, Array and phased array antenna basics. Chichester, West Sussex, England ; Hoboken, NJ: John Wiley \& Sons, 2005.

[31] A. de Hoop, The N-port Receiving Antenna and Its Equivalent Electrical Network. Philips Research Laboratories, 1975.

[32] H. Bui-Van, M. Arts, C. Craeye, and E. de Lera Acedo, "On the maximum absorbed power in receiving antenna arrays," IEEE Transactions on Antennas and Propagation, vol. 67, no. 3, pp. 1993-1995, Mar. 2019.

[33] C. G. Montgomery, R. H. Dicke, and E. M. Purcell, Principles of Microwave Engineering, ser. MIT Radiation Lab Series. New York: McGraw-Hill, 1948.

[34] J. T. Aberle, "Two-port representation of an antenna with application to non-foster matching networks," IEEE Trans. Antennas Propag., vol. 56, no. 5, pp. 1218-1222, May 2008.

[35] S. De Silva, L. Belostotski, and M. Okoniewski, "Radiation efficiency of an individual antenna in a system of multiple non-identical antennas," in General Assembly and Scientific Symposium of the International Union of Radio Science (URSI GASS), Aug 2017, pp. 1-4.

[36] S. De Silva, L. Belostotski, and M. Okoniewski, "Modeling and measuring of antenna array s-parameters and radiation efficiency," in IEEE International Symposium on Antennas and Propagation USNC/URSI National Radio Science Meeting, July 2017, pp. 2293-2294.

[37] R. Johnston and J. McRory, "An improved small antenna radiationefficiency measurement method," IEEE Antennas Propag. Mag., vol. 40, no. 5, pp. 40-48, Oct. 1998. 
[38] H. L. Thal, "A circuit model for spherical Wheeler cap measurements," IEEE Trans. Antennas Propag., vol. 59, no. 10, pp. 3638-3645, Oct. 2011.

[39] P. Groves, P. Conroy, L. Belostotski, and M. Okoniewski, "Measuring antenna noise parameters using a set of Wheeler caps," in Eur. Conf. Antennas Propag., Davos, Apr. 2016, pp. 1-4.

[40] P. Groves, P. Conroy, L. Belostotski, and M. Okoniewski, "Antenna twoport electrical and noise parameters," IEEE Antennas Wireless Propag. Lett., vol. 16, pp. 1265-1268, Nov. 2017.

[41] D. M. Pozar, Microwave engineering, 4th ed. Wiley, 2012.

[42] Symbolic math toolbox user's guide. Natick, MA: The MathWorks, Inc., 2016.

[43] Optimization toolbox user's guide. Natick, MA: The MathWorks, Inc., 2016.

[44] "IEEE standard definitions of terms for antennas," IEEE Std 145-2013 (Revision IEEE Std 145-1993), pp. 1-50, 2014.

[45] P. Russer, Electromagnetics, microwave circuit, and antenna design for communications engineering, ser. Artech House antennas and propagation library. Boston: Artech House, 2003.

[46] L. Belostotski, A. Sutinjo, R. H. Johnston, M. Okoniewski, M. A Petursson, and T. Burgess, "Study of thermal noise generated in a Vivaldi antenna using the improved Wheeler cap method," IEEE Antennas Wirel. Propag. Lett., vol. 10, no. 2, pp. 1047-1050, Sep. 2011.

[47] E. Newman, P. Bohley, and C. Walter, "Two methods for the measurement of antenna efficiency," IEEE Trans. Antennas Propag., vol. 23, no. 4, pp. 457-461, Jul. 1975.

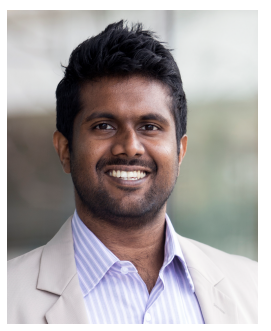

Supun De Silva is a passionate researcher in the field of electromagnetics, antenna arrays, mutual coupling, and RF circuits. He holds a master's degree in electrical engineering from the University of Calgary, Canada and a bachelor's degree in electrical engineering from the University of Moratuwa, Sri Lanka. He has co-authored several publications and presented at several conferences.

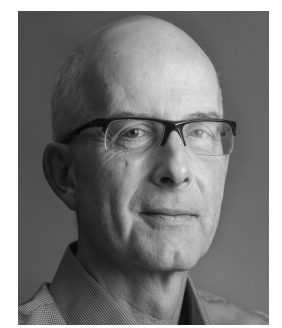

Michal Okoniewski is a Professor in the Electrical and Computer Engineering Department with Schulich School of Engineering, University of Calgary, Calgary, Canada. He is interested in many aspects of applied electromagnetics, ranging from computational electrodynamics, to tunable reflectarrays, transmittarrays, tunable and self configuring antennas, RF MEMS and RF micromachined devices. He is actively involved in bioelectromagnetics, where he works on tissue spectroscopy, imaging techniques, and micro-nuclear magnetic resonance spectroscopy. He has pioneered hardware acceleration of computational electromagnetic. He has co-founded two companies. Dr. Okoniewski has published 120 journal publications, and holds several patents.

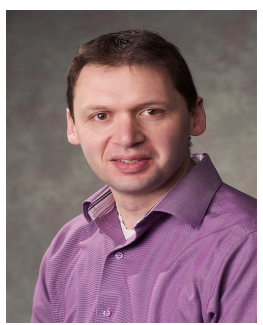

Leonid Belostotski (S'97-M'01-SM'14) received the B.Sc. and M.Sc. degrees in electrical engineering from the University of Alberta, Edmonton, AB,Canada, in 1997 and 2000, respectively, and the $\mathrm{Ph} . \mathrm{D}$. degree from the University of Calgary, Calgary, in 2007. He was an RF Engineer with Murandi Communications Ltd., Calgary, from 2001 to 2004. $\mathrm{He}$ is currently a Professor with the University of Calgary and the Canada Research Chair in HighSensitivity Radiometers and Receivers. His current research interests include RF and mixed-signal ICs, high sensitivity receiver systems, antenna arrays, and terahertz systems. He was a recipient of the IEEE Microwave Theory and Techniques-11 Contest on Creativity and Originality in Microwave Measurements in 2008 and the Outstanding Student Designer Award from Analog Devices, Inc., in 2007. He is the IEEE Southern Alberta Solid-State Circuits and Circuits and Systems Chapter's Chair. 